1 Hacettepe Journal of Mathematics and Statistics

$\bigcap$ Volume $44(2)$ (2015), 385-398

\title{
On soft continuous mappings and soft connectedness of soft topological spaces
}

\author{
Hai-Long Yang* ${ }^{*}$ Xiuwu Liao ${ }^{\dagger}$ and Sheng-Gang $\mathrm{Li}^{\ddagger}$
}

\begin{abstract}
The notion of soft topological space which is defined over an initial universe with a fixed set of parameters was introduced by Shabir and Naz. In this paper, the concept of soft continuous mapping between two soft topological spaces is first proposed. Then the main properties of soft continuous mappings are studied. Finally, the notion of soft connectedness of soft topological spaces is proposed, and some related properties are discussed.
\end{abstract}

2000 AMS Classification: 06F35.

Keywords: Soft sets, Soft topological spaces, Soft continuous mappings, Soft connectedness, Topological spaces.

Received 04 /10/2011 : Accepted 31 /01 /2013 Doi : 10.15672/HJMS.2015459876

\section{Introduction}

Uncertainty is an attribute of information. To solve the complicated problems in economics, engineering and environment, we cannot successfully use classical methods because of various uncertainties. A wide range of theories such as probability theory, fuzzy set theory, intuitionistic fuzzy set theory, rough set theory, vague set theory and the interval mathematics are well known and often useful mathematical approaches for modeling uncertainties. Each of these theories has its inherent difficulties as pointed out by Molodtsov [29]. The reason for these difficulties is, possibly, the inadequacy of the parametrization tool of the theories. Molodtsov [29] initiated the concept of soft set as a new mathematical tool for dealing with uncertainties that is free from the difficulties

${ }^{*}$ The School of Management, Xi'an Jiaotong University, Xi'an, 710049, PR China. Also College of Mathematics and Information Science, Shaanxi Normal University, Xi'an, 710119, PR China.

Email: yanghailong@snnu.edu.cn Corresponding Author.

†The School of Management, Xi'an Jiaotong University, Xi'an, 710049, PR China.

Email:liaoxiuwu@mail.xjtu.edu.cn

${ }^{\ddagger}$ College of Mathematics and Information Science, Shaanxi Normal University, Xi'an, 710119, PR China.

Email:topologyli@163.com 
that have troubled the existing theoretical approaches. This theory has proven useful in many fields such as decision making [8,14,26,32,38], data analysis [50], forecasting [44] and simulation [28].

The concept and basic properties of soft set theory were presented in $[29,33]$. In the classical soft set theory, a situation may be complex in the real world because of the fuzzy nature of the parameters. With this point of view, the classical soft sets have been extended to fuzzy soft sets [30,34], intuitionistic fuzzy soft sets [31], vague soft sets [46], interval-valued fuzzy soft sets [47] and interval-valued intuitionistic fuzzy soft sets [23].

Algebraic nature of soft sets has been studied by some authors. Maji et al. [33] presented some definitions on soft sets such as a soft subset, the complement of a soft set. Based on the analysis of several operations on soft sets introduced in [33], Ali et al. [3] presented some new algebraic operations for soft sets and proved that certain De Morgan's laws hold in soft set theory with respect to these new definitions. Qin and Hong introduced the concept of soft equality and some related properties were derived in [37]. Kharal and Ahmad [27] introduced the notion of a mapping on the classes of soft sets which is a pivotal notion for the advanced development of any new area of mathematical sciences. Babitha and Sunil [7] studied soft set relations and many related concepts were discussed. As a continuation of [7], kernels and closures of soft set relations, and soft set relation mappings were studied in [49]. In [39], Sezgin and Atagün presented a detailed theoretical study of operations on soft sets. Ali et al. [4] discussed algebraic structures of soft sets associated with new operations.

Up to the present, soft set theory has also been applied to several algebra structures: groups [1,2,40], semirings [12], rings [5,6,10], BCK/BCI-algebras [17-19], BCH-algebras [25], d-algebras [20], Hilbert algebras [21], ordered semigroups [22], BL-algebras [51] and fuzzy semigroup [48]. Xiao et al. [45] proposed the notions of exclusive disjunctive soft sets and studied some of its operations. Gong et al. [15] studied the bijective soft set with its operations. Ontology-based (or DL-based) soft set theory was presented in [24]. An idea of soft mappings is given and some of their properties are studied in [35]. Ge et al. [16] characterized some properties of topological spaces by using soft set theory. Recently, in [41], Shabir and Naz proposed the notion of soft topological space (defined over an initial universe with a fixed set of parameters) and investigated the basic properties. Tanay and Kandemir [42] studied topological structure of fuzzy soft sets. Çağman et al. defined the soft topology on a soft set, and presented its related properties in [9]. Shabir and Naz [41] pointed out that it will be necessary to carry out more theoretical research to establish a general framework for the practical application of soft topological spaces. In the present paper, we attempt to make some efforts in this aspect.

This paper will attempt to construct the basic theories about soft continuous mappings and soft connectedness of soft topological spaces. The rest of this paper is organized as follows. The next section briefly recalls the notions of soft set, topology and soft topology. In Section 3, based on soft set mapping, we define soft continuous mapping from one soft topological space to another soft topological space and give some equivalence characterizations of soft continuous mapping. Section 4 gives the concept of soft connectedness, and in Section 4, some related properties are discussed. The last section summarizes the conclusions and presents some topics for future research.

\section{Preliminaries}

In this section we will briefly recall the notions of soft set, topology and soft topology. See especially $[11,29,33,41]$ for further details and background. 
2.1. Definition. [29] Let $U$ be a common universe and $E$ be a set of parameters. Let $P(U)$ denote the power set of $U$ and $A \subset E$. A pair $(F, A)$ is called a soft set over $U$, where $F$ is a mapping given by $F: A \longrightarrow P(U)$.

In other words, a soft set over $U$ is a parameterized family of subsets of the universe $U$. For each $\varepsilon \in A, F(\varepsilon)$ may be considered as the set of $\varepsilon$-approximate elements of the soft set $(F, A)$.

2.2. Definition. [13] For two soft sets $(F, A)$ and $(G, B)$ over a common universe $U$ we say that $(F, A)$ is a soft subset of $(G, B)$ if

(1) $A \subset B$,

(2) $\forall \varepsilon \in A, F(\varepsilon) \subset G(\varepsilon)$.

We write $(F, A) \widetilde{\subset}(G, B)$.

$(F, A)$ is said to be a soft superset of $(G, B)$ if $(G, B)$ is a soft subset of $(F, A)$. We denote it by $(F, A) \widetilde{\supset}(G, B)$.

2.3. Remark. In [41], Shabir and Naz cited another notion of a soft subset as follows:

$(F, A)$ is a soft subset of $(G, B)$ iff (1) $A \subset B$ and (2) $\forall \varepsilon \in A, F(\varepsilon)$ and $G(\varepsilon)$ are identical approximations [33].

However, by the analysis of Definition 23, Theorem 1, Proposition 7 and Example 4 et al. in [41], all of these were obtained based on Definition 2.2 instead of the notion of a soft subset in [33]. Therefore, in the present paper, we will use the notion given in Definition 2.2 .

2.4. Definition. $[3,41]$ The relative complement of a soft set $(F, A)$ is denoted by $(F, A)^{\prime}$ and is defined by $(F, A)^{\prime}=\left(F^{\prime}, A\right)$ where $F^{\prime}: A \longrightarrow P(U)$ is a mapping given by $F^{\prime}(\alpha)=U-F(\alpha)$ for all $\alpha \in A$.

Obviously, $\left(F^{\prime}\right)^{\prime}=F$ and $\left((F, A)^{\prime}\right)^{\prime}=(F, A)$.

2.5. Definition. [33] A soft set $(F, A)$ over $U$ is said to be a NULL soft set and is denoted by $\Phi$, if $F(\varepsilon)=\emptyset$ for all $\varepsilon \in A$.

2.6. Definition. [33] The union of two soft sets $(F, A)$ and $(G, B)$ over a common universe $U$ is the soft set $(H, C)$, where $C=A \cup B$ and $\forall e \in C$,

$$
H(e)= \begin{cases}F(e), & \text { if } e \in A-B, \\ G(e), & \text { if } e \in B-A, \\ F(e) \cup G(e), & \text { if } e \in A \cap B .\end{cases}
$$

We write $(F, A) \cup(G, B)=(H, C)$.

2.7. Definition. [36] The intersection of two soft sets $(F, A)$ and $(G, B)$ over a common universe $U$ is the soft set $(H, C)$, where $C=A \cap B$ and $\forall e \in C, H(e)=F(e) \cap G(e)$.

We write $(F, A) \cap(G, B)=(H, C)$.

2.8. Proposition. Let $(F, A),(G, B)$ and $(H, C)$ be three soft sets over a common universe $U$. Then

(1) $(F, A) \cup((G, B) \cap(H, C))=((F, A) \cup(G, B)) \cap((F, A) \cup(H, C))$.

(2) $((F, A) \cap(G, B)) \cup(H, C)=((F, A) \cup(H, C)) \cap((G, B) \cup(H, C))$.

(3) $(F, A) \cap((G, B) \cup(H, C))=((F, A) \cap(G, B)) \cup((F, A) \cap(H, C))$.

(4) $((F, A) \cup(G, B)) \cap(H, C)=((F, A) \cap(H, C)) \cup((G, B) \cap(H, C))$.

(5) $(F, A) \cap((G, B) \cap(H, C))=((F, A) \cap(G, B)) \cap(H, C)$.

(6) $(F, A) \cup((G, B) \cup(H, C))=((F, A) \cup(G, B)) \cup(H, C)$.

Here the first five statements are from [36] and (6) is from [33]. It is easy to see that $(F, A) \cap\left(\bigcap_{i \in J}\left(G_{i}, B_{i}\right)\right)=\bigcap_{i \in J}\left((F, A) \cap\left(G_{i}, B_{i}\right)\right)$ and $(F, A) \cup\left(\bigcup_{i \in J}\left(G_{i}, B_{i}\right)\right)=$ $\bigcup_{i \in J}\left((F, A) \cup\left(G_{i}, B_{i}\right)\right)$, where $J$ is an index set, $(F, A)$ and $\left(G_{i}, B_{i}\right), i \in J$ are soft sets over a common universe $U$. 
2.9. Definition. [11,43] Let $X$ be an initial universe set and $\tau$ be the collection of subsets of $X$ then $\tau$ is said to be a topology on $X$ if

(1) $\emptyset, X$ belong to $\tau$,

(2) the union of any number of soft sets in $\tau$ belongs to $\tau$,

(3) the intersection of any two soft sets in $\tau$ belongs to $\tau$.

The pair $(X, \tau)$ is called a topological space. The members of $\tau$ are said to be open sets in $X$.

2.10. Definition. [11] (1) Let $(X, \tau)$ be a topological space. A subset $A$ of $X$ is said to be a closed set in $X$, if its complement $A^{\prime}$ belongs to $\tau$, where $A^{\prime}=X-A$.

(2) Let $\left(X, \tau_{1}\right),\left(Y, \tau_{2}\right)$ be two topological spaces and $f$ be a mapping from $X$ to $Y$. If $f^{-1}(B) \in \tau_{1}$ for all $B \in \tau_{2}$ then $f$ is called a continuous mapping from $\left(X, \tau_{1}\right)$ to $(Y$, $\left.\tau_{2}\right)$, where $f^{-1}(B)=\{x \in X \mid f(x) \in B\}$.

2.11. Proposition. [43] Let $(X, \tau)$ be a topological space. Then $(X, \tau)$ is connected if and only if there exists no $A, B \in \tau-\{\emptyset\}$ such that $A \cap B=\emptyset$ and $A \cup B=X$.

In the following, let $X$ be an initial universe set and $E$ be a non-empty set of parameters.

2.12. Definition. [41] Let $Y$ be a non-empty subset of $X$. Then $\tilde{Y}$ denotes the soft set $(Y, E)$ over $X$ for which $Y(\alpha)=Y$ for all $\alpha \in E$.

In particular, $(X, E)$ will be denoted by $\widetilde{X}$.

2.13. Definition. [41] Let $\tau$ be the collection of soft sets over $X$. Then $\tau$ is said to be a soft topology on $X$ if

(1) $\Phi, \widetilde{X}$ belong to $\tau$,

(2) the union of any number of soft sets in $\tau$ belongs to $\tau$,

(3) the intersection of any two soft sets in $\tau$ belongs to $\tau$.

The triple $(X, \tau, E)$ is called a soft topological space over $X$. The members of $\tau$ are said to be soft open sets in $X$.

2.14. Definition. [41] Let $(X, \tau, E)$ be a soft topological space over $X$. A soft set $(F, E)$ over $X$ is said to be a soft closed set in $X$, if its relative complement $(F, E)^{\prime}$ belongs to $\tau$.

2.15. Proposition. [41] Let $(X, \tau, E)$ be a soft topological space over $X$. Then the collection $\tau_{\alpha}=\{F(\alpha) \mid(F, E) \in \tau\}$ for each $\alpha \in E$, is a topology on $X$.

2.16. Definition. [41] Let $(X, \tau, E)$ be a soft topological space over $X$ and $(F, E)$ be a soft set over $X$. Then the soft closure of $(F, E)$, denoted by $\overline{(F, E)}$ is the intersection of all soft closed super sets of $(F, E)$.

$\overline{(F, E)}$ is the smallest soft closed set over $X$ which contains $(F, E)$ (see [41]).

2.17. Proposition. [41] Let $(X, \tau, E)$ be a soft topological space over $X$ and $(F, E),(G, E)$ be two soft sets over $X$. Then

(1) $\bar{\Phi}=\Phi, \overline{\widetilde{X}}=\tilde{X}$.

(2) $(F, E) \widetilde{\subset} \overline{(F, E)}$.

(3) $(F, E)$ is a soft closed set if and only if $(F, E)=\overline{(F, E)}$.

(4) $\overline{\overline{(F, E)}}=\overline{(F, E)}$.

(5) $(F, E) \widetilde{C}(G, E)$ implies $\overline{(F, E)} \widetilde{\subset} \overline{(G, E)}$. 


\section{Soft continuous mappings between soft topological spaces}

In this section, we will introduce the notion of soft continuous mapping between soft topological spaces and discuss some related properties. Let $X, Y$ be two initial universe sets and $E$ be a non-empty set of parameters. In what follows, the set of all soft sets over $X$ (resp., $Y$ ) will be denoted by $S(X)$ (resp., $S(Y)$ ).

In order to give the notion of soft continuous mapping, we first need to introduce the following notion of soft set mapping and inverse soft set mapping which can be regarded as special cases of Definitions 8 and 9 in the paper of Kharal and Ahmad [27], called "Mappings on soft classes".

3.1. Definition. Let $f$ be a mapping from $X$ to $Y$,

(1) The soft set mapping induced by $f$, denoted by the notation $f \rightarrow$, is a mapping from $S(X)$ to $S(Y)$ that maps $(F, E)$ to $f^{\rightarrow}((F, E))=\left(f^{\rightarrow}(F), E\right)$, where $f^{\rightarrow}(F)$ is defined by $f \rightarrow(F)(e)=\{f(x) \mid x \in F(e)\}, \forall e \in E$.

(2) The inverse soft set mapping induced by $f$, denoted by the notation $f^{\leftarrow}$, is a mapping from $S(Y)$ to $S(X)$ that maps $(G, E)$ to $f^{\leftarrow}((G, E))$, where $f^{\leftarrow}((G, E))=\left(f^{\leftarrow}(G), E\right)$ is defined by $f^{\leftarrow}(G)(e)=\{x \mid f(x) \in G(e)\}, \forall e \in E$.

3.2. Example. Let $X=\left\{h_{1}, h_{2}, h_{3}\right\}, Y=\left\{p_{1}, p_{2}\right\}$, and $E=\left\{e_{1}, e_{2}\right\}$. The mapping $f$ is given by $f\left(h_{1}\right)=p_{1}, f\left(h_{2}\right)=p_{1}, f\left(h_{3}\right)=p_{2}$.

(1) If $(F, E) \in S(X)$ is defined by $\left\{F\left(e_{1}\right)=\left\{h_{1}, h_{2}\right\}, F\left(e_{2}\right)=\left\{h_{1}, h_{3}\right\}\right\}$, then

$f^{\rightarrow}((F, E))=\left(f^{\rightarrow}(F), E\right)=\left\{f^{\rightarrow}(F)\left(e_{1}\right)=\left\{p_{1}\right\}, f^{\rightarrow}(F)\left(e_{2}\right)=Y\right\} \in S(Y)$.

(2) If $(G, B) \in S(Y)$ is defined by $\left\{G\left(e_{1}\right)=\left\{p_{2}\right\}, G\left(e_{2}\right)=\left\{p_{1}\right\}\right\}$, then

$f^{\leftarrow}((G, E))=\left(f^{\leftarrow}(G), E\right)=\left\{f^{\leftarrow}(G)\left(e_{1}\right)=\left\{h_{3}\right\}, f^{\leftarrow}(G)\left(e_{2}\right)=\left\{h_{1}, h_{2}\right\}\right\} \in S(X)$.

The following Propositions 3.3 and 3.4 give some basic properties of soft set mappings and inverse soft set mappings which can be regarded as special cases of Theorems 14 and 16 in [27].

3.3. Proposition. [27] Let $f$ be a mapping from $X$ to $Y,\left(F_{1}, E\right),\left(F_{2}, E\right) \in S(X)$. Then

(1) $f^{\rightarrow}(\Phi)=\Phi$.

(2) $\left(F_{1}, E\right) \widetilde{\subset}\left(F_{2}, E\right) \Longrightarrow f^{\rightarrow}\left(\left(F_{1}, E\right)\right) \widetilde{\subset} f^{\rightarrow}\left(\left(F_{2}, E\right)\right)$.

(3) $f^{\rightarrow}\left(\left(F_{1}, E\right) \cup\left(F_{2}, E\right)\right)=f^{\rightarrow}\left(\left(F_{1}, E\right)\right) \cup f^{\rightarrow}\left(\left(F_{2}, E\right)\right)$.

(4) $f \rightarrow\left(\left(F_{1}, E\right) \cap\left(F_{2}, E\right)\right) \widetilde{\subset} f \rightarrow\left(\left(F_{1}, E\right)\right) \cap f \rightarrow\left(\left(F_{2}, E\right)\right)$.

3.4. Proposition. [27] Let $f$ be a mapping from $X$ to $Y$ and $\left(G_{1}, E\right),\left(G_{2}, E\right) \in S(Y)$. Then

(1) $f^{\leftarrow}(\Phi)=\Phi, f^{\leftarrow}(\tilde{Y})=\tilde{X}$.

(2) $\left(G_{1}, E\right) \widetilde{\subset}\left(G_{2}, E\right) \Longrightarrow f^{\leftarrow}\left(\left(G_{1}, E\right)\right) \widetilde{\subset} f^{\leftarrow}\left(\left(G_{2}, E\right)\right)$.

(3) $f^{\leftarrow}\left(\left(G_{1}, E\right) \cup\left(G_{2}, E\right)\right)=f^{\leftarrow}\left(\left(G_{1}, E\right)\right) \cup f^{\leftarrow}\left(\left(G_{2}, E\right)\right)$.

(4) $f^{\leftarrow}\left(\left(G_{1}, E\right) \cap\left(G_{2}, E\right)\right)=f^{\leftarrow}\left(\left(G_{1}, E\right)\right) \cap f^{\leftarrow}\left(\left(G_{2}, E\right)\right)$.

(5) $f^{\leftarrow}\left(\left(G_{1}, E\right)^{\prime}\right)=\left(f^{\leftarrow}\left(\left(G_{1}, E\right)\right)\right)^{\prime}$.

3.5. Proposition. Let $f$ be a mapping from $X$ to $Y,(F, E) \in S(X),(G, E) \in S(Y)$. Then

(1) $f^{\leftarrow}\left(f^{\rightarrow}((F, E))\right) \widetilde{\supset}(F, E)$. If $f$ is one-one, then $f^{\leftarrow}\left(f^{\rightarrow}((F, E))\right)=(F, E)$.

(2) $f^{\rightarrow}\left(f^{\leftarrow}((G, E))\right) \widetilde{\subset}(G, E)$. If $f$ is surjective, then $f^{\rightarrow}\left(f^{\leftarrow}((G, E))\right)=(G, E)$.

Proof. (1) Let $f^{\rightarrow}((F, E))=(G, E)$. Then $\forall e \in E, f^{\leftarrow}(G)(e)=\{x \mid f(x) \in G(e)\}=$ $\{x \mid f(x) \in\{f(t) \mid t \in F(e)\}\} \supset F(e)$, which implies that $f^{\leftarrow}\left(f^{\rightarrow}((F, E))\right) \widetilde{\supset}(F, E)$.

If $f$ is one-one, notice that $\{x \mid f(x) \in\{f(t) \mid t \in F(e)\}\}=F(e)$, thus $f^{\leftarrow}\left(f^{\rightarrow}((F, E))\right)=$ $(F, E)$.

(2) Let $f^{\leftarrow}((G, E))=(F, E)$. Then $\forall e \in E, f^{\rightarrow}(F)(e)=\{f(x) \mid x \in F(e)\}=\{f(x) \mid$ $x \in\{t \mid f(t) \in G(e)\}\} \subset G(e)$, which implies that $f^{\rightarrow}\left(f^{\leftarrow}((G, E))\right) \widetilde{C}(G, E)$. 
If $f$ is surjective, notice that $\{f(x) \mid x \in\{t \mid f(t) \in G(e)\}\}=G(e)$, thus $f^{\rightarrow}\left(f^{\leftarrow}((G, E))\right)=(G, E)$.

3.6. Definition. Let $\left(X, \tau_{1}, E\right)$ and $\left(Y, \tau_{2}, E\right)$ be two soft topological spaces over $X$ and $Y$, respectively, and $f$ be a mapping from $X$ to $Y$. If $\forall(G, E) \in \tau_{2}$, we have $f^{\leftarrow}((G, E)) \in \tau_{1}$ then $f$ is called a soft continuous mapping from $\left(X, \tau_{1}, E\right)$ to $\left(Y, \tau_{2}, E\right)$.

Next, we will give an example about soft continuous mapping.

3.7. Example. Let $X=\left\{h_{1}, h_{2}, h_{3}\right\}, Y=\left\{p_{1}, p_{2}, p_{3}\right\}$ and $E=\left\{e_{1}, e_{2}\right\}$.

$\tau_{1}=\left\{\Phi, \widetilde{X},\left(F_{1}, E\right),\left(F_{2}, E\right)\right\}$, where $\left(F_{1}, E\right)$ and $\left(F_{2}, E\right)$ are two soft sets over $X$, defined as follows:

$F_{1}\left(e_{1}\right)=\left\{h_{2}\right\}, F_{1}\left(e_{2}\right)=\left\{h_{1}\right\}$,

$F_{2}\left(e_{1}\right)=\left\{h_{2}, h_{3}\right\}, F_{2}\left(e_{2}\right)=\left\{h_{1}, h_{2}\right\}$.

Then $\tau_{1}$ is a soft topology on $X$ and hence $\left(X, \tau_{1}, E\right)$ is a soft topological space over $X$. $\tau_{2}=\left\{\Phi, \tilde{Y},\left(G_{1}, E\right),\left(G_{2}, E\right)\right\}$, where $\left(G_{1}, E\right)$ and $\left(G_{2}, E\right)$ are two soft sets over $Y$, defined as follows:

$G_{1}\left(e_{1}\right)=\left\{p_{1}\right\}, G_{1}\left(e_{2}\right)=\left\{p_{2}\right\}$,

$G_{2}\left(e_{1}\right)=\left\{p_{1}, p_{3}\right\}, G_{2}\left(e_{2}\right)=\left\{p_{1}, p_{2}\right\}$.

Then $\tau_{2}$ is a soft topology on $Y$ and hence $\left(Y, \tau_{2}, E\right)$ is a soft topological space over $Y$. If $f$ is a mapping from $X$ to $Y$, defined as follows:

$f\left(h_{1}\right)=p_{2}, f\left(h_{2}\right)=p_{1}, f\left(h_{3}\right)=p_{3}$,

then it is easy to verify that $f^{\leftarrow}((G, E)) \in \tau_{1}$ for all $(G, E) \in \tau_{2}$. Thus $f$ is a soft continuous mapping from $\left(X, \tau_{1}, E\right)$ to $\left(Y, \tau_{2}, E\right)$.

3.8. Proposition. Let $\left(X, \tau_{1}, E\right)$ and $\left(Y, \tau_{2}, E\right)$ be two soft topological spaces over $X$ and $Y$, respectively. If $f$ is a soft continuous mapping from $\left(X, \tau_{1}, E\right)$ to $\left(Y, \tau_{2}, E\right)$, then $f$ is a continuous mapping from $\left(X,\left(\tau_{1}\right)_{\alpha}\right)$ to $\left(Y,\left(\tau_{2}\right)_{\alpha}\right)$ for all $\alpha \in E$.

Proof. By Proposition 2.15, $\left(X,\left(\tau_{2}\right)_{\alpha}\right)$ and $\left(Y,\left(\tau_{2}\right)_{\alpha}\right)$ are two topological spaces for all $\alpha \in E$. If $B \in\left(\tau_{2}\right)_{\alpha}$, then there exists a soft set $(G, E) \in \tau_{2}$ such that $B=G(\alpha)$. Since $f$ is a soft continuous mapping from $\left(X, \tau_{1}, E\right)$ to $\left(Y, \tau_{2}, E\right)$, then $f^{\leftarrow}((G, E)) \in \tau_{1}$. Thus $f^{-1}(B)=f^{-1}(G(\alpha))=\{x \mid f(x) \in G(\alpha)\}=f^{\leftarrow}(G)(\alpha) \in\left(\tau_{1}\right)_{\alpha}$,

and by Definition 2.10, $f$ is a continuous mapping from $\left(X,\left(\tau_{1}\right)_{\alpha}\right)$ to $\left(Y,\left(\tau_{2}\right)_{\alpha}\right)$.

Proposition 3.8 shows that a soft continuous mapping gives a parameterized family of continuous mappings.

3.9. Example. Let $\left(X, \tau_{1}, E\right)$ and $\left(Y, \tau_{2}, E\right)$ be two soft topological spaces and $f$ be the soft continuous mapping from $\left(X, \tau_{1}, E\right)$ to $\left(Y, \tau_{2}, E\right)$ given in Example 3.7. By Proposition 2.15,

$\left(\tau_{1}\right)_{e_{1}}=\left\{\emptyset, X,\left\{h_{2}\right\},\left\{h_{2}, h_{3}\right\}\right\}$ and $\left(\tau_{1}\right)_{e_{2}}=\left\{\emptyset, X,\left\{h_{1}\right\},\left\{h_{1}, h_{2}\right\}\right\}$ are two topologies on $X$, $Y$.

$\left(\tau_{2}\right)_{e_{1}}=\left\{\emptyset, Y,\left\{p_{1}\right\},\left\{p_{1}, p_{3}\right\}\right\}$ and $\left(\tau_{2}\right)_{e_{2}}=\left\{\emptyset, Y,\left\{p_{2}\right\},\left\{p_{1}, p_{2}\right\}\right\}$ are two topologies on

It is easy to verify that $f$ is a continuous mapping from $\left(X,\left(\tau_{1}\right)_{e_{1}}\right)$ to $\left(Y,\left(\tau_{2}\right)_{e_{1}}\right)$ and $f$ is also a continuous mapping from $\left(X,\left(\tau_{1}\right)_{e_{2}}\right)$ to $\left(Y,\left(\tau_{2}\right)_{e_{2}}\right)$.

Now we give an example to show that the inverse of Proposition 3.8 does not hold in general.

3.10. Example. Let $\left(X, \tau_{1}, E\right)$ be the soft topological space given in Example 3.7 and $Y=\left\{p_{1}, p_{2}, p_{3}\right\}, \tau_{2}=\left\{\Phi, \widetilde{Y},\left(G_{3}, E\right)\right\}$, where the soft set $\left(G_{3}, E\right)$ over $Y$ is defined by $\left\{G_{3}\left(e_{1}\right)=\left\{p_{1}\right\}, G_{3}\left(e_{2}\right)=\left\{p_{1}, p_{2}\right\}\right\}$. If $f$ is the mapping from $X$ to $Y$, given in Example 3.7 then by Proposition 2.15, 
$\left(\tau_{1}\right)_{e_{1}}=\left\{\emptyset, X,\left\{h_{2}\right\},\left\{h_{2}, h_{3}\right\}\right\}$ and and $\left(\tau_{1}\right)_{e_{2}}=\left\{\emptyset, X,\left\{h_{1}\right\},\left\{h_{1}, h_{2}\right\}\right\}$

$\left(\tau_{2}\right)_{e_{1}}=\left\{\emptyset, Y,\left\{p_{1}\right\}\right\}$ and $\left(\tau_{2}\right)_{e_{2}}=\left\{\emptyset, Y,\left\{p_{1}, p_{2}\right\}\right\}$ are two topologies on $Y$.

It can be easily seen that $f$ is a continuous mapping from $\left(X,\left(\tau_{1}\right)_{e_{1}}\right)$ to $\left(Y,\left(\tau_{2}\right)_{e_{1}}\right)$ continuous mapping from $\left(X,\left(\tau_{1}\right)_{e_{2}}\right)$ to $\left(Y,\left(\tau_{2}\right)_{e_{2}}\right)$. However,

$f^{\leftarrow}\left(\left(G_{3}, E\right)\right)=\left\{f^{\leftarrow}\left(G_{3}\right)\left(e_{1}\right)=\left\{h_{2}\right\}, f^{\leftarrow}\left(G_{3}\right)\left(e_{2}\right)=\left\{h_{1}, h_{2}\right\}\right\} \notin \tau_{1}$, which implies that $f$ is not a soft continuous mapping from $\left(X, \tau_{1}, E\right)$ to $\left(Y, \tau_{2}, E\right)$.

Next, we will give some equivalence characterizations of soft continuous mappings.

3.11. Proposition. Let $\left(X, \tau_{1}, E\right)$ (resp., $\left(Y, \tau_{2}, E\right)$ ) be a soft topological space over $X$ (resp., $Y$ ) and $f$ be a mapping from $X$ to $Y$. The following conditions are equivalent:

(1) $f$ is a soft continuous mapping from $\left(X, \tau_{1}, E\right)$ to $\left(Y, \tau_{2}, E\right)$.

(2) For each soft closed set $(G, E)$ in $Y, f^{\leftarrow}((G, E))$ is a soft closed set in $X$.

(3) For each soft set $(F, E)$ over $X, f^{\rightarrow}(\overline{(F, E)}) \widetilde{\subset} \overline{f \rightarrow((F, E))}$.

(4) For each soft set $(G, E)$ over $\left.Y, f^{\leftarrow} \overline{((G, E)}\right) \widetilde{\supset} \overline{f \leftarrow((G, E))}$.

Proof. (1) $\Longrightarrow(2)$ Let $(G, E)$ be a soft closed set in $Y$. Then $(G, E)^{\prime}$ be a soft open set in $Y$. By (1) and Proposition 3.4, $f^{\leftarrow}\left(\left(G_{1}, E\right)^{\prime}\right)=\left(f^{\leftarrow}\left(\left(G_{1}, E\right)\right)\right)^{\prime}$ is a soft open set in $X$. Hence $f^{\leftarrow}((G, E))$ is a soft closed set in $X$.

$(2) \Longrightarrow(3)$ Let $(F, E)$ be a soft set over $X$. By Proposition $2.17(2), f^{\rightarrow}((F, E))$ $\widetilde{\subset} \overline{f \rightarrow((F, E))}$. Then by Propositions $3.4(2)$ and 3.5 $(1),(F, E) \widetilde{\subset} f^{\leftarrow}\left(f^{\rightarrow}((F, E))\right) \widetilde{\subset} f^{\leftarrow}(\overline{f \rightarrow((F, E))})$. Since $\overline{f \rightarrow((F, E))}$ is a soft closed set in $Y$, then by $(2)$,

$f^{\leftarrow}(\overline{f \rightarrow((F, E))})$ is a soft closed set in $X$. Thus $\overline{(F, E)} \widetilde{\subset} f^{\leftarrow}(\overline{f \rightarrow((F, E))})$. Also by Propositions $3.3(2)$ and $3.5(2)$,

$f^{\rightarrow}(\overline{(F, E)}) \widetilde{\subset} f^{\rightarrow}\left(f^{\leftarrow}(\overline{f \rightarrow((F, E))})\right) \widetilde{\subset} \overline{f \rightarrow((F, E))}$. So $f^{\rightarrow(\overline{(F, E)})} \tilde{\subset} \overline{f \rightarrow((F, E))}$.

$(3) \Longrightarrow(4)$ Let $(G, E)$ be a soft set over $Y$. By (3), Propositions $3.5(2)$ and $2.17(5)$, $f^{\rightarrow}(\overline{(f \leftarrow((G, E))}) \widetilde{\subset} \overline{f \rightarrow(f \leftarrow((G, E)))} \widetilde{\subset} \overline{(G, E)}$. Then by Propositions 3.4 (2) and 3.5 (1), $\left.f^{\leftarrow} \overline{((G, E)}\right) \widetilde{\supset} f^{\leftarrow}\left(f^{\rightarrow}(\overline{f \leftarrow((G, E))})\right) \widetilde{\supset} \overline{f \leftarrow((G, E))}$.

$(4) \Longrightarrow(1)$ If $(G, E)$ is a soft open set in $Y$, then $(G, E)^{\prime}$ is a soft closed set in $Y$. By (4) and Proposition $2.17(3), \overline{f^{\leftarrow}\left((G, E)^{\prime}\right)} \widetilde{\subset} f^{\leftarrow}\left((G, E)^{\prime}\right)$. Obviously, $\overline{f^{\leftarrow}\left((G, E)^{\prime}\right)} \widetilde{\supset} f^{\leftarrow}\left((G, E)^{\prime}\right)$. Thus $\overline{f^{\leftarrow}\left((G, E)^{\prime}\right)}=f^{\leftarrow}\left((G, E)^{\prime}\right)$, which implies that $f^{\leftarrow}\left((G, E)^{\prime}\right)=\left(f^{\leftarrow}((G, E))\right)^{\prime}$ (by Proposition $3.4(5))$ is a soft closed set in $X$. Therefore, $f^{\leftarrow}((G, E))$ is a soft open set in $X$. So $f$ is a soft continuous mapping from $\left(X, \tau_{1}, E\right)$ to $\left(Y, \tau_{2}, E\right)$.

\section{Soft connectedness of soft topological spaces}

In this section, we will introduce the notion of soft connectedness of soft topological spaces and discuss some related properties.

4.1. Definition. Let $(X, \tau, E)$ be a soft topological space over $X$. If there exists no $(F, E),(G, E) \in \tau-\{\Phi\}$ such that $(F, E) \cap(G, E)=\Phi$ and $(F, E) \cup(G, E)=\tilde{X}$, then $(X, \tau, E)$ is called soft connected.

4.2. Definition. [41] Let $X$ be an initial universe set, $E$ be a set of parameters and $\tau=\{\Phi, \tilde{X}\}$. Then $\tau$ is called the soft indiscrete topology on $X$ and $(X, \tau, E)$ is said to be the soft indiscrete space over $X$.

4.3. Example. Any soft indiscrete space is soft connected.

4.4. Example. Let $X=\left\{h_{1}, h_{2}, h_{3}\right\}$ and $E=\left\{e_{1}, e_{2}\right\} . \tau=\left\{\Phi, \widetilde{X},\left(F_{1}, E\right),\left(F_{2}, E\right)\right.$, $\left.\left(F_{3}, E\right)\right\}$, where $\left(F_{1}, E\right),\left(F_{2}, E\right)$, and $\left(F_{3}, E\right)$ are three soft sets over $X$, defined as follows:

$F_{1}\left(e_{1}\right)=\left\{h_{2}\right\}, F_{1}\left(e_{2}\right)=\emptyset$

$F_{2}\left(e_{1}\right)=\left\{h_{1}, h_{3}\right\}, F_{2}\left(e_{2}\right)=\emptyset$, 


$$
F_{3}\left(e_{1}\right)=X, F_{3}\left(e_{2}\right)=\emptyset .
$$

Then $(X, \tau, E)$ is a soft topological space over $X$. It is easy to verify that there exists no $(F, E),(G, E) \in \tau-\{\Phi\}$ such that $(F, E) \cap(G, E)=\Phi$ and $(F, E) \cup(G, E)=\tilde{X}$, so $(X, \tau, E)$ is soft connected. By the definition of $\tau_{\alpha}$, we have $\tau_{e_{1}}=\left\{\emptyset, X,\left\{h_{2}\right\},\left\{h_{1}, h_{3}\right\}\right\}$, and $\tau_{e_{2}}=\{\emptyset, X\}$. Obviously, $\left(X, \tau_{e_{1}}\right)$ is not connected, but $\left(X, \tau_{e_{2}}\right)$ is connected.

It is worth pointing out that this example shows that $(X, \tau, E)$ is soft connected but $\left(X, \tau_{\alpha}\right)(\alpha \in E)$ may not be connected.

4.5. Remark. $(X, \tau, E)$ may not be soft connected even if $\left(X, \tau_{\alpha}\right)$ is connected for every parameter $\alpha \in E$.

4.6. Example. Let $X$ be a non-empty initial universe set and $E=\left\{e_{1}, e_{2}\right\}$ be the set of parameters. $\tau=\{\Phi, \widetilde{X},(F, E),(G, E)\}$, where $(F, E)$ and $(G, E)$ are two soft sets over $X$, defined as follows:

$$
\begin{aligned}
& F\left(e_{1}\right)=\emptyset, F\left(e_{2}\right)=X, \\
& G\left(e_{1}\right)=X, G\left(e_{2}\right)=\emptyset .
\end{aligned}
$$

Then $(X, \tau, E)$ is a soft topological space over $X$. Obviously, $(F, E),(G, E) \in \tau-\{\Phi\}$, $(F, E) \cap(G, E)=\Phi$ and $(F, E) \cup(G, E)=\widetilde{X}$, so $(X, \tau, E)$ is not soft connected. However, $\tau_{e_{1}}=\tau_{e_{2}}=\{\emptyset, X\}$ (indiscrete topology), which implies that $\left(X, \tau_{e_{1}}\right)$ and $\left(X, \tau_{e_{2}}\right)$ are connected.

The following proposition gives some equivalence characterizations of soft connectedness.

4.7. Proposition. Let $(X, \tau, E)$ be a soft topological space over $X$. Then the following conditions are equivalent:

(1) $(X, \tau, E)$ is soft connected.

(2) There exists no $(F, E),(G, E) \in \tau^{\prime}-\{\Phi\}$ such that $(F, E) \cap(G, E)=\Phi$ and $(F, E) \cup$

$(G, E)=\widetilde{X}$, where $\tau^{\prime}=\left\{(F, E)^{\prime} \mid(F, E) \in \tau\right\}$.

(3) There exists no $(F, E),(G, E) \in S(X)-\{\Phi\}$ such that $((F, E) \cap \overline{(G, E)}) \cup(\overline{(F, E)} \cap$

$(G, E))=\Phi$ and $(F, E) \cup(G, E)=\widetilde{X}$.

(4) There exists no $(F, E) \in S(X)-\{\Phi, \widetilde{X}\}$ such that $(F, E) \in \tau \cap \tau^{\prime}$.

Proof. (1) $\Longrightarrow(2)$ Assume there exist $(F, E),(G, E) \in \tau^{\prime}-\{\Phi\}$ such that $(F, E) \cap$ $(G, E)=\Phi$ and $(F, E) \cup(G, E)=\widetilde{X}$. Then $\forall e \in E, F(e) \cap G(e)=\emptyset$ and $F(e) \cup G(e)=X$. Thus $\forall e \in E, F^{\prime}(e)=X-F(e)=G(e)$ and $G^{\prime}(e)=X-G(e)=F(e)$, which implies that $(F, E)^{\prime}=(G, E) \in \tau^{\prime}-\{\Phi\}$ and $(G, E)^{\prime}=(F, E) \in \tau^{\prime}-\{\Phi\}$. Then there exist $(F, E),(G, E) \in \tau-\{\Phi\}$ such that $(F, E) \cap(G, E)=\Phi$ and $(F, E) \cup(G, E)=\widetilde{X}$. However, $(X, \tau, E)$ is soft connected. This is a contradiction.

$(2) \Longrightarrow(3)$ Assume there exist $(F, E),(G, E) \in S(X)-\{\Phi\}$ such that $((F, E) \cap$ $\overline{(G, E)}) \cup(\overline{(F, E)} \cap(G, E))=\Phi$ and $(F, E) \cup(G, E)=\tilde{X}$. Obviously, $(F, E) \cap(G, E)=\Phi$. By Propositions 2.8 and 2.17, $\overline{(G, E)}=\overline{(G, E)} \cap \widetilde{X}=\overline{(G, E)} \cap((F, E) \cup(G, E))=$ $\overline{((G, E)} \cap(F, E)) \cup(\overline{(G, E)} \cap(G, E))=(G, E)$, which implies that $(G, E)$ is a soft closed set. By using the same methods, we can show that $(F, E)$ is also a soft closed set. Hence there exist $(F, E),(G, E) \in \tau^{\prime}-\{\Phi\}$ such that $(F, E) \cap(G, E)=\Phi$ and $(F, E) \cup(G, E)=\tilde{X}$. This is a contradiction. So (3) holds.

$(3) \Longrightarrow(4)$ Assume there exists $(F, E) \in \tau \cap \tau^{\prime}-\{\Phi, \tilde{X}\}$. If take $(G, E)=(F, E)^{\prime}$ then $(F, E),(G, E) \in \tau \cap \tau^{\prime}-\{\Phi\}(\subset S(X)-\{\Phi\})$. Besides, we have $((F, E) \cap \overline{(G, E)}) \cup$ $(\overline{(F, E)} \cap(G, E))=(F, E) \cap(G, E)=\Phi$ and $(F, E) \cup(G, E)=\widetilde{X}$. This is a contradiction. So (4) holds.

$(4) \Longrightarrow(1)$ Assume $(X, \tau, E)$ is not soft connected. Then there exist $(F, E),(G, E)$ $\in \tau-\{\Phi\}$ such that $(F, E) \cap(G, E)=\Phi$ and $(F, E) \cup(G, E)=\tilde{X}$. It is easy to see that 
$(F, E)^{\prime}=(G, E)$ and $(G, E)^{\prime}=(F, E)$. Thus $(F, E),(G, E) \in \tau \cap \tau^{\prime}-\{\Phi, \tilde{X}\}$. This is a contradiction.

4.8. Definition. (1) The difference $(H, E)$ of two soft sets $(F, E)$ and $(G, E)$ over $X$, denoted by $(F, E)-(G, E)$ (or $(F, E) \backslash(G, E))$ is defined as $H(e)=F(e)-G(e)$ for all $e \in E$.

(2) Let $(F, E)$ be a soft set over $X$ and $Y$ be a non-empty subset of $X$. Then the soft subset of $(F, E)$ over $Y$ denoted by $\left({ }^{Y} F, E\right)$ is defined as ${ }^{Y} F(e)=Y \cap F(e)$, for all $e \in E$. In other words $\left({ }^{Y} F, E\right)=\tilde{Y} \cap(F, E)$.

(3) Let $(X, \tau, E)$ be a soft topological space over $X$ and $Y$ be a non-empty subset of $X$. Then $\tau_{Y}=\left\{\left({ }^{Y} F, E\right) \mid(F, E) \in \tau\right\}$ is said to be the soft relative topology on $Y$ and $\left(Y, \tau_{Y}, E\right)$ is called a soft subspace of $(X, \tau, E)$.

In fact, $\tau_{Y}$ is a soft topology on $Y$.

4.9. Proposition. Let $\left(Y, \tau_{Y}, E\right)$ be a soft subspace of a soft topological space $(X, \tau, E)$. If $\left(Z,\left(\tau_{Y}\right)_{Z}, E\right)$ is a soft subspace of $\left(Y, \tau_{Y}, E\right)$ then $\left(Z,\left(\tau_{Y}\right)_{Z}, E\right)$ is also a soft subspace of $(X, \tau, E)$.

Proof. $\left(\tau_{Y}\right)_{Z}=\{\tilde{Y} \cap(F, E) \mid(F, E) \in \tau\}_{Z}$

$$
\begin{aligned}
& =\{\widetilde{Z} \cap \tilde{Y} \cap(F, E) \mid(F, E) \in \tau\} \\
& =\{\widetilde{Z} \cap(F, E) \mid(F, E) \in \tau\} \\
& =\tau_{Z} .
\end{aligned}
$$

So $\left(Z,\left(\tau_{Y}\right)_{Z}, E\right)$ is a soft subspace of $(X, \tau, E)$.

4.10. Definition. Let $(X, \tau, E)$ be a soft topological space over $X$ and $Y$ be a non-empty subset of $X$. If $\left(Y, \tau_{Y}, E\right)$ is soft connected then $Y$ is called a soft connected subset of $X$.

Let $\left(Y, \tau_{Y}, E\right)$ be a soft subspace of a soft topological space $(X, \tau, E)$. For a soft set $(F, E) \in S(Y), \overline{(F, E)}$ and $\overline{(F, E)}_{Y}$ will denote the soft closures of $(F, E)$ in $(X, \tau, E)$ and $\left(Y, \tau_{Y}, E\right)$, respectively.

Now we discuss some basic properties about soft connectedness.

4.11. Proposition. Let $(X, \tau, E)$ be a soft topological space over $X$. If $Y$ is a soft connected subset of $X$, then there exists no $(F, E),(G, E) \in \tau-\{\Phi\}$ such that $(F, E) \cap$ $(G, E)=\Phi$ and $(F, E) \cup(G, E)=\tilde{Y}$.

Proof. If there exist $(F, E),(G, E) \in \tau-\{\Phi\}$ such that $(F, E) \cap(G, E)=\Phi$ and $(F, E) \cup$ $(G, E)=\tilde{Y}$, then $(F, E)=\widetilde{Y} \cap(F, E) \in \tau_{Y}-\{\Phi\}$ and $(G, E)=\tilde{Y} \cap(G, E) \in \tau_{Y}-\{\Phi\}$.

However, $Y$ is a soft connected subset of $X$. This is a contradiction.

The following example shows that the inverse of Proposition 4.11 may not hold in general:

4.12. Example. Let $X=\{a, b, c\}, Y=\{b, c\}$, and $E=\left\{e_{1}, e_{2}\right\} . \tau=\left\{\Phi, \widetilde{X},\left(F_{1}, E\right)\right.$, $\left.\left(F_{2}, E\right),\left(F_{3}, E\right)\right\}$, where $\left(F_{1}, E\right),\left(F_{2}, E\right)$, and $\left(F_{3}, E\right)$ are three soft sets over $X$, defined as follows:

$F_{1}\left(e_{1}\right)=\{a, b\}, F_{1}\left(e_{2}\right)=\{a, c\}$,

$F_{2}\left(e_{1}\right)=\{a, c\}, F_{2}\left(e_{2}\right)=\{a, b\}$,

$F_{3}\left(e_{1}\right)=\{a\}, F_{3}\left(e_{2}\right)=\{a\}$.

Then $(X, \tau, E)$ is a soft topological space over $X$.

By Definition 4.8, we have

${ }^{Y}\left(F_{1}, E\right)=\left\{{ }^{Y} F_{1}\left(e_{1}\right)=\{b\},{ }^{Y} F_{1}\left(e_{2}\right)=\{c\}\right\}$,

${ }^{Y}\left(F_{2}, E\right)=\left\{{ }^{Y} F_{2}\left(e_{1}\right)=\{c\},{ }^{Y} F_{2}\left(e_{2}\right)=\{b\}\right\}$,

${ }^{Y}\left(F_{3}, E\right)=\left\{{ }^{Y} F_{3}\left(e_{1}\right)=\emptyset,{ }^{Y} F_{3}\left(e_{2}\right)=\emptyset\right\}=\Phi$. 
Then $\tau_{Y}=\left\{\Phi, \tilde{Y},{ }^{Y}\left(F_{1}, E\right),{ }^{Y}\left(F_{2}, E\right)\right\}$.

It is easy to verify that there exists no $(F, E),(G, E) \in \tau-\{\Phi\}$ such that $(F, E) \cap$ $(G, E)=\Phi$ and $(F, E) \cup(G, E)=\tilde{Y}$. However, ${ }^{Y}\left(F_{1}, E\right),{ }^{Y}\left(F_{2}, E\right) \in \tau_{Y}-\{\Phi\},{ }^{Y}\left(F_{1}, E\right) \cap$ ${ }^{Y}\left(F_{2}, E\right)=\Phi$ and ${ }^{Y}\left(F_{1}, E\right) \cup{ }^{Y}\left(F_{2}, E\right)=\tilde{Y}$, i.e. $\left(Y, \tau_{Y}, E\right)$ is not soft connected.

By Proposition 4.11, we can easily show the following corollary:

4.13. Corollary. Let $(X, \tau, E)$ be a soft topological space over $X$ and $Y$ be a soft connected subset of $X$. If there exist $(F, E),(G, E) \in \tau$ such that $(F, E) \cap(G, E)=\Phi$ and $\widetilde{Y} \widetilde{C}(F, E) \cup(G, E)$ then $\widetilde{Y} \widetilde{C}(F, E)$ or $\widetilde{Y} \widetilde{C}(G, E)$.

4.14. Lemma. [41] Let $\left(Y, \tau_{Y}, E\right)$ be a soft subspace of a soft topological space $(X, \tau, E)$ and $(F, E)$ be a soft set over $X$, then $(F, E)$ is soft closed in $Y$ if and only if $(F, E)=$ $\widetilde{Y} \cap(G, E)$ for some soft closed set $(G, E)$ in $X$.

4.15. Proposition. Let $(X, \tau, E)$ be a soft topological space over $X$ and $Y$ be a nonempty subset of $X$. Then $Y$ is a soft connected subset of $X$ if and only if there exists no $(F, E),(G, E) \in S(Y)-\{\Phi\}$ such that $((F, E) \cap \overline{(G, E)}) \cup(\overline{(F, E)} \cap(G, E))=\Phi$ and $(F, E) \cup(G, E)=\widetilde{Y}$.

Proof. For all $(F, E),(G, E) \in S(Y)-\{\Phi\}$, by Definition 2.16, Proposition 2.8 and Lemma 4.14, we have

$$
\begin{aligned}
(F, E) \cap{\overline{(G, E)_{Y}}}_{Y} & =(F, E) \cap\left(\bigcap\left\{(H, E) \mid(H, E) \in\left(\tau_{Y}\right)^{\prime},(H, E) \widetilde{\supset}(G, E)\right\}\right)^{\S} \\
& =(F, E) \cap\left(\bigcap\left\{\widetilde{Y} \cap(Q, E) \mid(Q, E) \in \tau^{\prime},(Q, E) \widetilde{\supset}(G, E)\right\}\right)^{\uparrow} \\
& =((F, E) \cap \widetilde{Y}) \cap\left(\bigcap\left\{(Q, E) \mid(Q, E) \in \tau^{\prime},(Q, E) \widetilde{\supset}(G, E)\right\}\right) \\
& =(F, E) \cap\left(\bigcap\left\{(Q, E) \mid(Q, E) \in \tau^{\prime},(Q, E) \widetilde{\supset}(G, E)\right\}\right) \\
& =(F, E) \cap \overline{(G, E) .}
\end{aligned}
$$

Similarly, we can show that $\overline{(F, E)}_{Y} \cap(G, E)=\overline{(F, E)} \cap(G, E)$.

By Proposition 4.7, $\left(Y, \tau_{Y}, E\right)$ is soft connected if and only if there exists no $(F, E),(G, E) \in$ $S(Y)-\{\Phi\}$ such that $\left((F, E) \cap \overline{(G, E)}_{Y}\right) \cup\left(\overline{(F, E)}_{Y} \cap(G, E)\right)=\Phi$ and $(F, E) \cup(G, E)=\tilde{Y}$. Then $\left(Y, \tau_{Y}, E\right)$ is soft connected if and only if there exists no $(F, E),(G, E) \in S(Y)-\{\Phi\}$ such that $((F, E) \cap \overline{(G, E)}) \cup(\overline{(F, E)} \cap(G, E))=\Phi$ and $(F, E) \cup(G, E)=\widetilde{Y}$, i.e. $Y$ is a soft connected subset of $X$ if and only if there exists no $(F, E),(G, E) \in S(Y)-\{\Phi\}$ such that $((F, E) \cap \overline{(G, E)}) \cup(\overline{(F, E)} \cap(G, E))=\Phi$ and $(F, E) \cup(G, E)=\tilde{Y}$.

4.16. Proposition. Let $(X, \tau, E)$ be a soft topological space over $X$ and $Y$ be a soft connected subset of $X$. If there exist $(F, E),(G, E) \in S(Y)$ such that $((F, E) \cap \overline{(G, E)}) \cup$ $(\overline{(F, E)} \cap(G, E))=\Phi$ and $\tilde{Y} \widetilde{C}(F, E) \cup(G, E)$ then $\widetilde{Y} \widetilde{C}(F, E)$ or $\tilde{Y} \widetilde{C}(G, E)$.

Proof. If there exist $(F, E),(G, E) \in S(Y)$ such that $((F, E) \cap \overline{(G, E)}) \cup(\overline{(F, E)} \cap(G, E))=$ $\Phi$ and $\widetilde{Y} \widetilde{C}(F, E) \cup(G, E)$ then by Propositions 2.8 and 2.17 , we have

$((\widetilde{Y} \cap(F, E)) \cap \overline{(\widetilde{Y} \cap(G, E))}) \cup \overline{((\widetilde{Y} \cap(F, E))} \cap(\tilde{Y} \cap(G, E)))$

$\widetilde{C}((\widetilde{Y} \cap(F, E)) \cap \overline{(G, E)}) \cup \overline{(\overline{(F, E)}} \cap(\widetilde{Y} \cap(G, E)))$

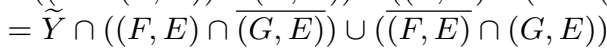

$=\tilde{Y} \cap \Phi$

$=\Phi$.

Besides, we have $\tilde{Y} \cap(F, E), \tilde{Y} \cap(G, E) \in S(Y)$, and $(\tilde{Y} \cap(F, E)) \cup(\tilde{Y} \cap(G, E))=$ $\widetilde{Y} \cap((F, E) \cup(G, E))=\tilde{Y}$. Since $Y$ is a soft connected subset of $X, \widetilde{Y} \cap(F, E)=\Phi$ or $\widetilde{Y} \cap(G, E)=\Phi$ by Proposition 4.15. If $\widetilde{Y} \cap(F, E)=\Phi$ then by $(\widetilde{Y} \cap(F, E)) \cup(\widetilde{Y} \cap(G, E))=$ $\widetilde{Y}$, we have $\widetilde{Y} \widetilde{C}(G, E)$. Similarly, if $\widetilde{Y} \cap(G, E)=\Phi$ then $\widetilde{Y} \widetilde{C}(F, E)$.

\footnotetext{
${ }^{\S}\left(\tau_{Y}\right)^{\prime}=\left\{\widetilde{Y}-(W, E) \mid(W, E) \in \tau_{Y}\right\}$.

$\boldsymbol{\pi}^{\prime}=\{\tilde{X}-(V, E) \mid(V, E) \in \tau\}$.
} 
4.17. Proposition. Let $(X, \tau, E)$ be a soft topological space over $X, Y$ be a soft connected subset of $X$ and $Z$ be a non-empty subset of $X$. If $\widetilde{Y} \widetilde{\subset} \widetilde{Z} \widetilde{\widetilde{Y}}$ then $Z$ is also a soft connected subset of $X$.

Proof. Assume that $Z$ is not a soft connected subset of $X$. By Proposition 4.15, there exist $(F, E),(G, E) \in S(Z)-\{\Phi\}(\subset S(Y))$ such that $((F, E) \cap \overline{(G, E)}) \cup(\overline{(F, E)} \cap(G, E))=$ $\Phi$ and $(F, E) \cup(G, E)=\widetilde{Z}$. Then $\widetilde{Y} \widetilde{C}(F, E) \cup(G, E)$. Since $Y$ is a soft connected subset of $X$, we have $\widetilde{Y} \widetilde{C}(F, E)$ or $\widetilde{Y} \widetilde{C}(G, E)$ by Proposition 4.16. If $\widetilde{Y} \widetilde{\subset}(F, E)$, then $\widetilde{Z} \cap(G, E) \widetilde{\subset} \overline{(F, E)} \cap(G, E)=\Phi$ for $\widetilde{Z} \widetilde{\subset} \widetilde{\widetilde{Y}} \widetilde{\subset} \overline{(F, E)}$. Thus $(G, E)=\widetilde{Z} \cap(G, E)=\Phi$. This is a contradiction. Similarly, if $\widetilde{Y} \widetilde{C}(G, E)$ then $(F, E)=\Phi$. This is also a contradiction.

The following proposition shows that the soft connectedness is an invariant property under a soft continuous mapping.

4.18. Proposition. Let $f$ be a soft continuous mapping from soft topological space $\left(X, \tau_{1}, E\right)$ to soft topological space $\left(Y, \tau_{2}, E\right)$. If $\left(X, \tau_{1}, E\right)$ is soft connected and $f(X) \neq \emptyset$ then $f(X)$ is a soft connected subset of $Y$.

Proof. Assume $f(X)$ is not a soft connected subset of $Y$. By Proposition 4.15, there exist $(F, E),(G, E) \in S(f(X))-\{\Phi\}(\subset S(Y)-\{\Phi\})$ such that $((F, E) \cap \overline{(G, E)}) \cup(\overline{(F, E)} \cap$ $(G, E))=\Phi$ and $(F, E) \cup(G, E)=\widehat{f(X)}$. Then $f^{\leftarrow}((F, E)), f^{\leftarrow}((G, E)) \in S(X)-\{\Phi\}$ and by Propositions 3.11 and 3.4 ,

$$
\begin{aligned}
&\left(f^{\leftarrow}((F, E)) \cap \overline{\left.f^{\leftarrow((G, E)}\right)}\right) \cup\left(\overline{\left.f^{\leftarrow((F, E)}\right)} \cap f^{\leftarrow}((G, E))\right) \\
& \widetilde{C}\left(f^{\leftarrow}((F, E)) \cap f^{\leftarrow}(\overline{(G, E)})\right) \cup\left(f^{\leftarrow}(\overline{(F, E)}) \cap f^{\leftarrow}((G, E))\right)
\end{aligned}
$$$$
=f^{\leftarrow}(((F, E) \cap \overline{(G, E)}) \cup(\overline{(F, E)} \cap(G, E)))
$$

$=f^{\leftarrow}(\Phi)$

$=\Phi$.

Besides, by Propositions 3.4, and 3.5, we have

$f^{\leftarrow}((F, E)) \cup f^{\leftarrow}((G, E))=f^{\leftarrow}((F, E) \cup(G, E))=f^{\leftarrow} \widetilde{(f(X))}=f^{\leftarrow}(f \rightarrow(\widetilde{X}))=\widetilde{X}$.

It follows that $\left(X, \tau_{1}, E\right)$ is not soft connected. This is a contradiction. So $f(X)$ is a soft connected subset of $Y$.

To illustrate the idea of Proposition 4.18, we give the following example:

4.19. Example. Let $X=\left\{h_{1}, h_{2}, h_{3}\right\}, Y=\left\{p_{1}, p_{2}, p_{3}\right\}$ and $E=\left\{e_{1}, e_{2}\right\}$. $\tau_{1}=$ $\left\{\Phi, \widetilde{X},\left(F_{1}, E\right),\left(F_{2}, E\right)\right\}$, where $\left(F_{1}, E\right)$ and $\left(F_{2}, E\right)$ are two soft sets over $X$, defined as $F_{1}\left(e_{1}\right)=\left\{h_{2}\right\}, F_{1}\left(e_{2}\right)=\emptyset, F_{2}\left(e_{1}\right)=\left\{h_{1}, h_{3}\right\}, F_{2}\left(e_{2}\right)=\emptyset$. Then $\left(X, \tau_{1}, E\right)$ is a soft topological space over $X$ and it is easy to verify that $\left(X, \tau_{1}, E\right)$ is soft connected.

Let $\tau_{2}=\{\Phi, \tilde{Y},(G, E)\}$, where $(G, E)=\left\{G\left(e_{1}\right)=\left\{p_{1}\right\}, G\left(e_{2}\right)=\left\{p_{2}, p_{3}\right\}\right\}$. Then $\left(Y, \tau_{2}, E\right)$ is a soft topological space over $Y$.

If $f: X \longrightarrow Y$ is a mapping defined as $f\left(h_{1}\right)=f\left(h_{2}\right)=f\left(h_{3}\right)=p_{1}$, obviously, $f$ is a soft continuous mapping from $\left(X, \tau_{1}, E\right)$ to $\left(Y, \tau_{2}, E\right)$. In this case, $f(X)=\left\{p_{1}\right\} \neq \emptyset$ and it is easy to verify that $f(X)$ is a soft connected subset of $Y$.

\section{Conclusion}

In this paper, we construct the basic theories about soft continuous mappings and soft connectedness of soft topological spaces. We also give some examples to explain these new notions. Moreover the concepts and some results proposed in this paper can be extended into fuzzy cases. In our future study on soft topological spaces, may be the following topics should be considered:

(1) To discuss soft basis, soft sub-basis of soft topological spaces, 
(2) To study soft compactness of soft topological spaces,

(3) To describe the real world application of soft topological spaces.

\section{Acknowledgements}

The authors are very grateful to the referees for their valuable comments and suggestions for improving this paper.

This work was supported by the National Natural Science Foundation of China (No. 61473181), China Postdoctoral Science Foundation funded project (No. 2013M532063) and Shaanxi Province Postdoctoral Science Foundation funded project (The first batch).

\section{References}

[1] Aktaş, H. and Çağman, N. Soft sets and soft groups, Information Science 177 (13), 2726$2735,2007$.

[2] Aygunoglu, A. and Aygun, H. Introduction to fuzzy soft groups, Computers and Mathematics with Applications 58 (6), 1279-1286, 2009.

[3] Ali, M.I., Feng, F., Liu, X., Min, W.K. and Shabir, M. On some new operations in soft set theory, Computers and Mathematics with Applications 57 (9), 1547-1553, 2009.

[4] Ali, M.I., Shabir, M. and Naz, M. Algebraic structures of soft sets associated with new operations, Computers and Mathematics with Applications 61 (9), 2647-2654, 2011.

[5] Acar, U., Koyuncu, F. and Tanay, B. Soft sets and soft rings, Computers and Mathematics with Applications 59 (11), 3458-3463, 2010.

[6] Atagün, A.O. and Sezgin, A. Soft substructures of rings, fields and modules, Computers and Mathematics with Applications 61 (3), 592-601, 2011.

[7] Babitha, K.V. and Sunil, J.J. Soft set relations and functions, Computers and Mathematics with Applications 60 (7), 1840-1849, 2010.

[8] Çağman, N. and Enginoğlu, S. Soft matrix theory and its decision making, Computers and Mathematics with Applications 59 (10), 3308-3314, 2010.

[9] Çă̆man, N., Karataş, S. and Enginoglu, S. Soft topology, Computers and Mathematics with Applications 62 (1), 351-358, 2011.

[10] Çelik, Y., Ekiz C. and Yamak, S. A new view on soft rings, Hacettepe Journal of Mathematics and Statistics 40 (2), 273-286, 2011.

[11] Engelking, R. General Topology, Panstwowe Wydawnictwo Naukowe, 1975.

[12] Feng, F., Jun, Y.B. and Zhao, X.Z. Soft semirings, Computers and Mathematics with Applications 56, 2621-2628, 2008.

[13] Feng, F., Li, C., Davvaz, B. and Ali, M.I. Soft sets combined with fuzzy sets and rough sets: a tentative approach, Soft Computing 14, 8999-9911, 2010.

[14] Feng, F., Jun, Y.B., Liu, X. and Li, L. An adjustable approach to fuzzy soft set based decision making, J. Comput. Appl. Math. 234 (1), 10-20, 2010.

[15] Gong, K., Xiao, Z. and Zhang, X. The bijective soft set with its operations, Computers and Mathematics with Applications 60 (8), 2270-2278, 2010.

[16] Ge, X., Li, Z. and Ge, Y. Topological spaces and soft sets, Journal of Computational Analysis and Applications 13 (5), 881-885, 2011.

[17] Jun, Y.B. Soft BCK/BCI-algebras, Computers and Mathematics with Applications 56 (5), 1408-1413, 2008.

[18] Jun, Y.B. and Park, C.H. Applications of soft sets in ideal theory of BCK/BCI-algebras, Information Science 178 (11), 2466-2475, 2008.

[19] Jun, Y.B., Lee, K.J. and Zhan, J. Soft p-ideals of soft BCI-algebras, Computers and Mathematics with Applications 58 (10), 2060-2068, 2009.

[20] Jun, Y.B., Lee, K.J. and Park, C.H. Soft set theory applied to ideals in d-algebras, Computers and Mathematics with Applications 57 (3), 367-378, 2009.

[21] Jun, Y.B. and Park, C.H. Applications of soft sets in Hilbert algebras, Iranian Journal of Fuzzy Systems 6 (2), 75-86, 2009.

[22] Jun, Y.B., Lee, K.J. and Khan, A. Soft ordered semigroups, Math. Logic Quart. 56 (1), $42-50,2010$ 
[23] Jiang, Y., Tang, Y., Chen, Q., Liu, H. and Tang, J. Interval-valued intuitionistic fuzzy soft sets and their properties, Computers and Mathematics with Applications 60 (3), 906-918, 2010.

[24] Jiang, Y., Tang, Y., Chen, Q., Wang, J. and Tang, S. Extending soft sets with description logics, Computers and Mathematics with Applications 59 (6), 2087-2096, 2010.

[25] Kazancı O., Yılaz, Ş and Yamak, S. Soft sets and soft BCH-algebras, Hacettepe Journal of Mathematics and Statistics 39 (2), 205-217, 2010.

[26] Kong, Z., Gao, L. and Wang, L. Comment on "A fuzzy soft set theoretic approach to decision making problems", J. Comput. Appl. Math. 223 (2), 540-542, 2009.

[27] Kharal, A. and Ahmad, B. Mappings on fuzzy soft classes, Advances in Fuzzy Systems, 2009, 1-6, 2009.

[28] Kalayathankal, S.J. and Singh, G.S. A fuzzy soft flood alarm model, Math. Comput. Simul. 80 (5), 887-893, 2010.

[29] Molodtsov, D. Soft set theory - first results, Computers and Mathematics with Applications 37, 19-31, 1999.

[30] Maji, P.K., Biswas, R. and Roy, A.R. Fuzzy soft sets, J. Fuzzy Math. 9 (3), 589-602, 2001.

[31] Maji, P.K., Biswas, R. and Roy, A.R. Intuitionistic fuzzy soft sets, J. Fuzzy Math. 9 (3), 677-692, 2001.

[32] Maji, P.K., Roy, A.R. and Biswas, R. An application of soft sets in a decision making problem, Computers and Mathematics with Applications 44 (8-9), 1077-1083, 2002.

[33] Maji, P.K., Biswas, R. and Roy, A.R. Soft set theory, Computers and Mathematics with Applications 45, 555-562, 2003.

[34] Majumdar, P. and Samanta, S.K. Generalised fuzzy soft sets, Computers and Mathematics with Applications 59 (4), 1425-1432, 2010

[35] Majumdar, P. and Samanta, S.K. On soft mappings, Computers and Mathematics with Applications 60 (9), 2666-2672, 2010.

[36] Pei, D. and Miao, D. From soft sets to information systems, in: Granular Computing, IEEE International Conference, 2, 617-621, 2005.

[37] Qin, K. and Hong, Z. On soft equality, J. Comput. Appl. Math. 234 (5), 1347-1355, 2010.

[38] Roy, A.R. and Maji, P.K. A fuzzy soft set theoretic approach to decision making problems, J. Comput. Appl. Math. 203 (2), 412-418, 2007.

[39] Sezgin, A. and Atagün, A.O. On operations of soft sets, Computers and Mathematics with Applications 61, 1457-1467, 2011.

[40] Sezgin, A. and Atagün, A.O. Soft groups and normalistic soft groups, Computers and Mathematics with Applications 62 (2), 685-698, 2011.

[41] Shabir, M. and Naz, M. On soft topological spaces, Computers and Mathematics with Applications 61, 1786-1799, 2011.

[42] Tanay, B. and Kandemir, M.B. Topological structure of fuzzy soft sets, Computers and Mathematics with Applications 61 (10), 2952-2957, 2011.

[43] Xiong, J.C. Point set topology teaching materials, Higher Education Press, 2001 (in Chinese).

[44] Xiao, Z., Gong, K. and Zou, Y. A combined forecasting approach based on fuzzy soft sets, J. Comput. Appl. Math. 228 (1), 326-333, 2009.

[45] Xiao, Z., Gong, K., Xia, S. and Zou, Y. Exclusive disjunctive soft sets, Computers and Mathematics with Applications 59 (6), 2128-2137, 2010.

[46] $\mathrm{Xu}, \mathrm{W} ., \mathrm{Ma}, \mathrm{J} .$, Wang, S. and Hao, G. Vague soft sets and their properties, Computers and Mathematics with Applications 59 (2), 787-794, 2010.

[47] Yang, X.B., Lin, T.Y., Yang, J.Y., Li, Y. and Yu, D. Combination of interval-valued fuzzy set and soft set, Computers and Mathematics with Applications 58 (3), 521-527, 2009.

[48] Yang, C.-F. Fuzzy soft semigroups and fuzzy soft ideals, Computers and Mathematics with Applications 61 (2), 255-261, 2011

[49] Yang, H.-L. and Guo, Z.-L. Kernels and closures of soft set relations, and soft set relation mappings, Computers and Mathematics with Applications 61 (3), 651-662, 2011.

[50] Zou, Y. and Xiao, Z. Data analysis approaches of soft sets under incomplete information, Knowledge-Based Systems 21 (8), 941-945, 2008. 
[51] Zhan, J. and Jun, Y.B. Soft BL-algebras based on fuzzy sets, Computers and Mathematics with Applications 59 (6), 2037-2046, 2010. 